\title{
Banker, Spekulanten und Abzocker
}

Hans Heinrich Brunner

Korrespondenz:

Dr. med.

Hans Heinrich Brunner

Seestrasse

CH-6354 Vitznau

\section{Liebe Spitzen-Banker, Spekulanten und Abzocker}

Es ist nun wirklich an der Zeit, dass die Ärztinnen und Ärzte Ihnen ihren tief empfundenen Dank aussprechen. Sie haben uns aus der medialen Schusslinie gebracht, in der wir unserer Einkommen wegen während Jahrzehnten Angriff über Angriff über uns ergehen lassen mussten und dauernd gelitten haben.

Sie haben die Geldmesslatte im öffentlichen Bewusstsein in solch schwindelerregende Höhen gebracht, dass selbst ein Chefarzteinkommen von 2 Millionen heute ein Nicht-Thema wäre. Dies konditional formuliert, weil es solches schon lange nicht mehr gibt.

Und welche Nichtigkeit ist ein Grundversorgereinkommen von Fr. 120000.- vor Steuern neben Boni von hoch zweistelliger Millionenhöhe von Spitzenbankern - wobei sich dieser Begriff entsprechend der Philosophie Ihres Gewerbes nach Höhe des Einkommens und nicht nach Kompetenz und Professionalität definieren muss. Solche Ärzteeinkommen erregen schon das Mitleid linker Gewerkschafter. In der Tat muss sich die Ärzteschaft ernsthaft überlegen, ob sie ihre Einkommenspolitik nicht in die Hände des VPODs legen will. Die sind in der politischen Dampferzeugung viel versierter als wir Ärztinnen und Ärzte, die auf dem Bundeshausplatz stehen, in höflichem Ton einige Forderungen stellen und dann auch nicht wissen, was es nun weiter soll.

Sie haben es natürlich bemerkt: Hier schwingt neben Dank auch Neid mit. Nicht nur wegen der astronomischen Differenzen zwischen Boni und medizinischen Spitzeneinkommen, sondern auch wegen der Nonchalance, mit der Sie es fertigbringen, völlig unprofessionell ein System fast in den Super-Gau zu bringen und dann, von allen Gerichten unbelangt, weil nicht einmal belangbar, auf den weichen Kissen Ihrer ebenso märchenhaften Abgangsentschädigungen weich gebettet aus sicherer Distanz zwischen 9 Löchern auf dem Golf Course und der Happy Hour am Oberen Zürichsee gelassenen Blickes die Entwicklung des von Ihnen angerichteten Chaos zu verfolgen. Wissend, dass alle Androhungen von Strafverfahren nichts anderes als linke Wahlpropaganda sind. Im Vergleich: Jeder Chefarzt mit gleichen Nichtqualifikationen würde längere Zeit im Zuchthaus verbringen und nachfolgend zur lebenslangen Grabpflege aller seiner Opfer verurteilt werden. Nichts von Otium cum dignitate und Happy Hour. Nur Verzehr des Peculiums.

Neben Neid und Frustration derjenigen, die auch zum Heloten-Heer derjenigen zählen, die die von Ihnen kunstvoll angerichtete Speise als Steuerzahler nicht nur hinunterwürgen, sondern auch noch über Steuern, denen wir nicht leichtfüssig entgehen wie Sie, über wahrscheinliche Inflation und anderes mehr, bezahlen müssen, ist es - natürlich haben Sie auch dies bemerkt - stille Bewunderung, die uns bewegt. Nach der alten Weisheit, dass man Frustration am besten dadurch Herr wird, dass ihr Nützliches abgewonnen wird, komme ich zum Vorschlag folgender Massnahmen, die zu realisieren ich meine Standesorganisation einlade:

Wir organisieren zusammen mit «pensionierten» Spitzenbankern Weiter- und Fortbildungskurse - natürlich mit Credits - in denen folgende Inhalte vermittelt werden:

- Wie schaffe ich es, völlig unprofessionell zu arbeiten, immensen Schaden anzurichten, straffrei zu bleiben und ein finanziell gesichertes Otium cum dignitate zu verleben? Bemerkung: Es muss ja nicht unbedingt am rechten oder oberen Zürichseeufer sein.

- Gibt es eine Möglichkeit, Ärzteeinkommen in Boni zu verwandeln?

- Wie kann ich alle Forderungen nach Qualität in den Wind schlagen und nach einer durch mein unprofessionelles Tun ausgelösten Katastrophe weiter agieren und verdienen wie zuvor?

- (In Klausur): Wie bringe ich es als Ärztin oder Arzt fertig, ein schwindelerregendes Einkommen und ein ebenso atemberaubendes Vermögen zu haben und doch nur Steuern zu bezahlen, die mich knapp an die Grenzen der Sozialhilfe bringen?

Ich gehe davon aus, dass die angesprochenen Cracks nicht honoriert werden wollen. Sie werden solche Aktivitäten im Rahmen mildtätiger Caritas sehen, mit der Sie auch Urner Bergbauern, die Heilsarmee und gefallene Frauen beglücken. Die hier denkbaren Honorare hätten ohnehin Trinkgeldcharakter; Sie nehmen keine Trinkgelder, sondern lassen solche gnädig fallen.

In der Hoffnung, dass Sie diese Zeilen wohlwollend empfangen, und in der bestimmten Erwartung, dass meine Standesorganisation nun aktiv wird, verbleibe ich

mit hochachtungsvollen Grüssen

Dr. med. Hans Heinrich Brunner, Alt-Präsident der FMH Verbindung der Schweizer Ärztinnen und Ärzte 